\title{
GENETIC METHODS FOR DETECTING ASTROCYTES, NEURONS AND NEUROGENESIS
}

Natalia Nikolaevna Shusharina ${ }^{1}$, Ekaterina Vladimirovna Silina ${ }^{2}$, Aleksandr Alexandrovich Vasilyev ${ }^{1}$, Irina Nikolaevna Dominova ${ }^{1}$, Victor Aleksandrovich Stupin ${ }^{3}$, Tatiana Georgievna Sinelnicova ${ }^{2}$, Egor Borisovich Sotnikov ${ }^{1}$, Andrey Victorivich Turkin ${ }^{1}$, Maksim Vladimirovich Patrushev ${ }^{1}$

${ }^{1}$ Institute of Life Sciences; Immanuel Kant Baltic Federal University (IKBFU), Kaliningrad, Moscow, Russia

${ }^{2}$ Department of Human Pathology, I.M. Sechenov First Moscow State Medical University (Sechenov University), Moscow, Russia

${ }^{3}$ Department of hospital surgery №1; N.I. Pirogov Russian National Research Medical University (RNRMU), Moscow, Russia

\section{GENETIČKE METODE DETEKCIJE ASTROCITA,} NEURONA I NEUROGENEZE

\author{
Natalia Nikolaevna Shusharina ${ }^{1}$, Ekaterina Vladimirovna Silina ${ }^{2}$, Aleksandr Alexandrovich Vasilyev ${ }^{1}$, Irina Nikolaevna Dominova ${ }^{1}$, Victor Aleksandrovich Stupin ${ }^{3}$, \\ Tatiana Georgievna Sinelnicova², Egor Borisovich Sotnikov ${ }^{1}$, Andrey Victorivich Turkin ${ }^{1}$, Maksim Vladimirovich Patrushev $^{1}$ \\ ${ }^{1}$ Institut za nauku Immanuel Kant, Balticki drzavni univerzitet (IKBFU), Kaliningrad, Moskva, Rusija \\ ${ }^{2}$ Odsek za humanu patologiju, I.M. Sechenov Prvi Moskovski državni medicinski univerzitet (Univerzitet Sechenov), Moskva, Rusija \\ ${ }^{3}$ Odsek za hirurgiju №1; N.I. Pirogov Ruski nacionalni istraživački medicinski univerzitet (RNRMU), Moskva, Rusija
}

\begin{abstract}
Two sets of reactants for modelling neurogenesis (SRMN) were developed based on the designed and tested genetic structures of lentiviral vectors. SRMN-1 contains the genetic construct LVV-GFAP-GCaMP3 and is intended for cellspecific transduction in astroglia cells. SRMN-2 contains the genetic construct LVV-PRSX8-TN-XXL and is intended for the phenotype-specific transduction in neurons. The present study examined SRMN-1 and SRMN-2 samples and assessed their efficiency in vitro and in vivo in Norvegicus rats. Specificity to particular cell types for all SRMN samples exceeded 97\%. The number of induced signalling cascades was determined via activation of intracellular ingsignalling cascades in neurons and astrocytes (purinergic receptors and $\beta$-adrenoceptors). The results demonstrated dynamic recording of fluorescent signals and a two-fold increase in intensity after addition of the activator in all samples. The experimental SRMN samples revealed successful and stable transfection of catecholaminergic neurons and astrocytes, data on transfection efficiency, specificity of the developed genetic structures of SRMN, and calcium dynamics in transfected neurons and astrocytes.

These results confirm the crucial role of astrocytes in ensuring neurogenesis. The results in pure cell culture (in vitro) were identical to the in vivo results in animals.
\end{abstract}

Keywords: neurogenesis astrocytes, neurons, signalling cascades, genetic structure, viral vector.

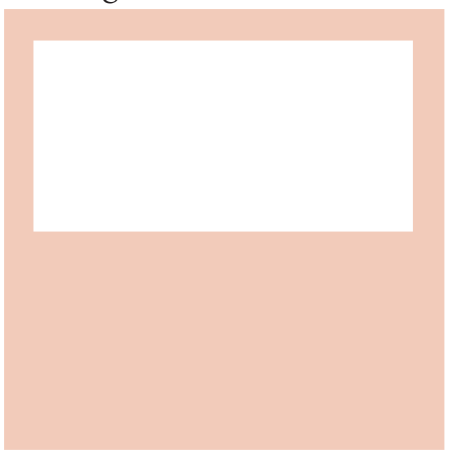




\section{INTRODUCTION}

The incidence of cerebral pathology has progressively increased in recent decades because of the increasing life expectancy of the human population. This increase especially applies to disabling pathologies, such as apoplexy and neurodegenerative diseases $(1,2)$. One possible reason for the lack of significant progress in treatment may be that the primary vector of investigations of the pathogenesis of neurological diseases is neurons. This focus may be due to the ambiguous effects of neuroprotective therapy (3).

Glia represent greater than $50 \%$ of cells in the brain, and these cells outnumber neurons (4). Astrocytes are the most common and important glial cells, account for 30$40 \%$ of the total number of brain cells (5). Astrocytes primarily form the haematoencephalic barrier and provide an adequate supply of nutrition to neurons. These functions are disrupted in Parkinson's disease (6). Astrocytes provide structural and metabolic support, regulate synaptic transmission and water transport, and play an active role neuronal plasticity $(7,8)$. Therefore, astrocytes may underlie diseases of the central nervous system.

The development of genetic engineering and genetics in neurosciences yielded data that confirmed this assumption. For example, scientists examined the expression levels of known key genes of Parkinson's disease in human and mouse astrocytes and neurons and found that monogenic mutations were expressed in astrocytes at levels comparable to or, in some cases, higher than neurons $(9,10)$. New scientific evidence confirmed the important roles of astrocytes in the development of various diseases, including neurodegeneration $(11,12)$. However, this role of astrocytes remains controversial in scientific circles.

Fundamental research in this area is required to resolve this controversy, and genetic methods of research appear promising.

The present study developed genetic methods of registering and correcting neurogenesis disruptions in the brain using viral vectors and assessed the efficiency of the developed methods in vitro and in vivo.

\section{MATERIALS AND METHODS}

The first experiments used two sets of reactants for modelling neurogenesis (SRMN) based on the designed and tested genetic structures.

The set of reactants for modelling neurogenesis No. 1 (SRMN-1) contained the genetic construct LVV-GFAPGCaMP3 based on the lentiviral vector, which is specific for astroglia. These constructs were maintained in DMEM cultural medium with $10 \%$ foetal bovine serum. SRMN-1 carries a genetically encoded calcium indicator (GCaMP3) under the control of cell-specific promoter of glial fibrillar acid protein (GFAP), which is intended for cell-specific transduction in astroglia cells (Fig. 1).
A set of reactants for modelling neurogenesis No. 2 (SRMN-2) contained the genetic construct LVV-PRSx8TN-XXL based on the lentiviral vector, which is specific for catecholaminergic neurons. These cells were maintained in DMEM culture medium with 10\% foetal bovine serum. SRMN-2 is intended for phenotype-specific transduction in neurons (Fig. 2).

These genetic structures exhibit the following characteristics: lentiviral vector; visualized chemically; size of the experimental sample not greater than 25,000 nucleotides/ pairs of nucleotides; auxiliary components of genetic constructs not greater than 370 nucleotides/pairs of nucleotides; specificity to certain cell type not less than $97 \%$; only transferred cells exhibited differentiation (SRMN No. 1 for astrocytes, SRMN No. 2 for catecholaminergic neurons); and the titer of the genetic constructs not less than $0.5 \times 10^{6}$ T.U./ml (T.U. - transducing unit).

The SRMN exhibited the following primary properties: (1) ability to integrate into the genome of the target cell to ensure permanent expression of the transgene; (2) ability to transfect differentiated cells; (3) wide tropism due to packing of vesicular stomatitis into the shell of the virus; and (4) low immunogenicity when used in animals.

The developed methods of registration and correction of neurogenesis abnormalities in the brain based on functional and/or genetically coded sensors included the following technological procedures:

1. Preparation of fragments for cloning (amplification of fragment of post-transcriptional regulatory element, amplification of fragment of marker gene, amplification of cell-specific promoter);

2. Cloning fragments into a vector (selecting target fragments in the DNA sequence, fractionation of fragments by the method of gel electrophoresis and purification, cloning of purified fragments into a vector);

3. Producing vectors (cultivation of HEK293T cell culture, preparation of the vector for transfection, seeding cells into Petri dishes, transfection, vector production, isolation and purification of the vector); and

4. Concentrating the vector (concentrating vectors via centrifugation, storage), filling, labelling and packaging.

The second stage of work performed experimental studies using the developed SRMN samples, followed by assessment of their efficiency, in vitro and in vivo in 24 rats Rattus Norvegicus.

Each experiment examined 3 samples of SRMN-1 and 3 samples of SRMN-2.

The experimental design for samples of SRMN to assess efficiency in the in vitro experiments was based on a combination of methods for isolating and cultivating astrocytes and neurons in the brain, their transfection with viral constructs, and recording the fluorescent images for subsequent assessments of performance.

The model was pure cultures of astrocytes and neurons to assess the efficiency of genetic structures because fluorescent protein expression (GCaMP3 and TN-XXL) 


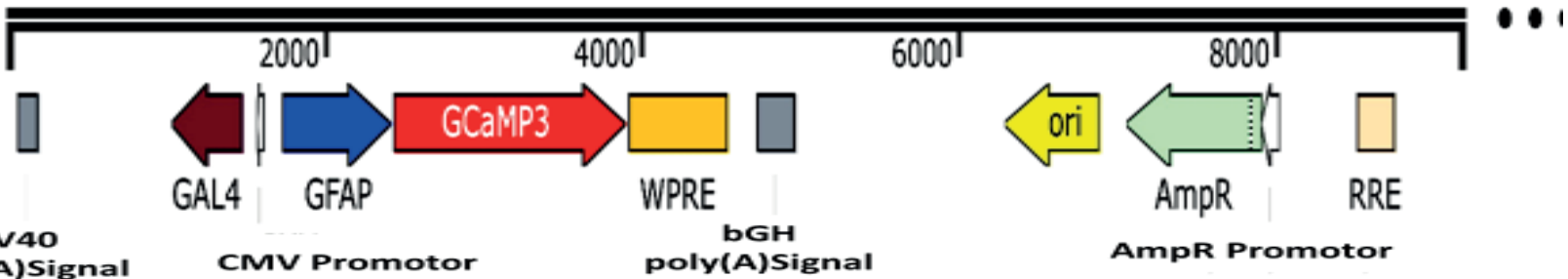
poly(A)Signal CMV Promotor

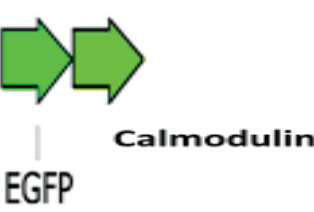

AmpR Promotor

Figure 1. The diagram of the genetic construct LVV-GFAP-GCaMP3 carrying genetically encoded calcium indicator GCaMP3 under the control of cell-specific promoter glial fibrillose acidic protein (GFAP): SV40 poly(A) signal is a signal of SV40 polyadenitis formation; GAL4 is a chimeric transactivator that includes parts of transactivatorg domain of mice protein NF- $\mathrm{kBp} 65$ fused with a DNA-binding domain of yeast protein GAL4; CMV is the minimal promoter of human cytomegalovirus; GFAP is the compact promoter of glial fibrillary acidic protein; GCaMP3 is a sequence that encodes genetically encoded calcium indicator GCaMP3; EGFP is reinforced green fluorescent protein; Calmodulin is a calcium binding protein; WPRE is a post-transcription regulatory element of Groundhog hepatitis B virus; bGHpoly(A) signal is a signal of polyadenylation; ori is the high-scale start point of ColE1/pMB1/pBR322/pUC plasmids replication; AmpR is a sequence that provides resistance to ampiciline, carbencillin, and other bonded antibiotics; AmpR promoter is a promoter that ensures resistance to ampicillin; bom is the basic cell region of the plasmid pBR322; RRE is the rev-responsive element of HIV-1, which provides a Rev-responsible export of mRNA from the nucleus into the cytoplasm

could only be analysed qualitatively and quantitatively in the monolayer cell cultures. The detailed protocols for all stages of this work were developed.

The following protocol was used to obtain pure cultures of neurons and astrocytes: obtaining a mixed suspension and mixed culture of brain cells from rats; and purification to obtain pure cultures of neurons and astrocytes. Cell cultures were further investigated using SRMN-1 and SRMN-2 to assess specificity and identify induced cascades (Figure 3).

Cell cultures were transfected in standard growth medium with the addition of an aliquot of sample SRMN to neurons or astrocytes. Laser scanning confocal microscopy was used to assess the efficiency of SRMN samples. This method allowed the recording of fluorescent protein sensor expression (GCaMP3 and TN-XXL) with high precision.

Specificity of genetic constructs to certain cell types was determined via in vitro transfection of pure rat brain cell cultures, staining for cell-specific markers, and record- ing of the fluorescent signal using an LSM 780 confocal microscope (Carl Zeiss).

Astrocyte primary culture was obtained from 3-dayold rats. The cells were grown in the Dulbecco modified Eagle's medium (DMEM) (with $4.5 \mathrm{~g} / \mathrm{l}$ glucose, glutamine, and pyruvate) ("GIBCO", UK) with $10 \%$ foetal bovine serum (“GIBCO”, UK).

Neuronal primary cultures were obtained from 1-dayold rats. The cerebral cortex was extracted on ice in KrebsRinger solution $(135 \mathrm{mM}$ of $\mathrm{NaCl}, 5 \mathrm{mM}$ of $\mathrm{KCl}, 1 \mathrm{mM}$ of $\mathrm{MgSO}_{4}, 0.4 \mathrm{mM}$ of $\mathrm{KH}_{2} \mathrm{PO} 4,15 \mathrm{mM}$ of glucose, and 20 $\mathrm{mM}$ of HEPES, pH 7.4), trypsinized in a $0.08 \%$ solution of trypsin-EDTA for $10 \mathrm{~min}$ at $37^{\circ} \mathrm{C}$, and ground in a $0.008 \%$ solution DNAaza-I containing $0.05 \%$ of a trypsin inhibitor. Cells were resuspended in minimal Eagle's medium with Earl's salts (BME) containing 10\% of heat-inactivated foetal bovine serum ("GIBCO”, Germany), $2 \mathrm{mM}$ of GlutaMAX-I, and gentamicin $(100 \mu \mathrm{g} / \mathrm{ml})$. Cells were seeded on 24-hole tablets covered with poly-L-lysine at a density

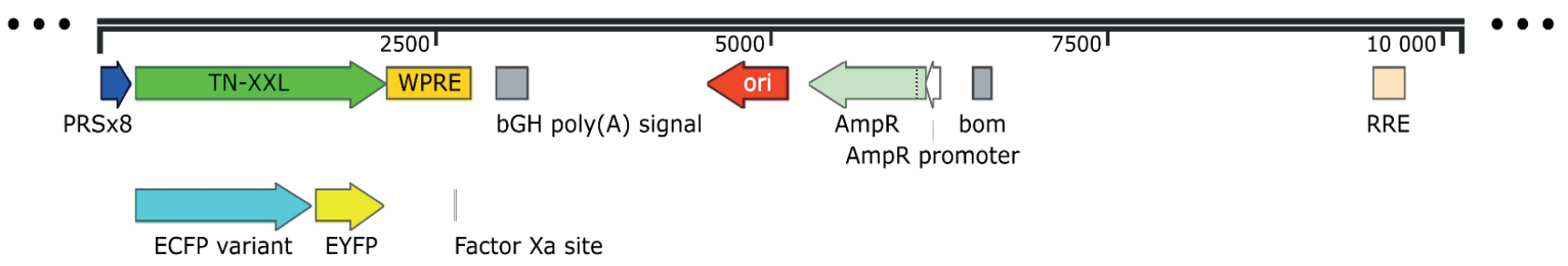

Figure 2. The diagram of genetic construct LVV-PRSx8-TN-XL, TN-XXL under the control of artificial specific neuronal promoter (PRSx8): PRSx8 is an artificial phenotype-specific neuronal promoter; TN-XXL is a sequence that encodes the genetically coded calcium indicator TN-XXL; ECFP variant is enhanced blue fluorescent protein; EYFP is enhanced yellow fluorescent protein; WPRE is post-regulatory element of the Groundhog hepatitis B virus; bGH poly(A) signal is a polyadenylation signal; ori is the high-scale point of plasmids ColE1/pMB1/pBR322/pUC replication start; AmpR is the sequence that ensures resistance to ampicillin, carbenicillin, and other bonded antibiotics; AmpR promoter is a promoter that ensures resistance to ampicillin; bom is the basic cell region of the plasmid pBR322; RRE is the rev-responsive element of HIV-1, which provides a Rev-responsible export of mRNA from the nucleus into the cytoplasm 


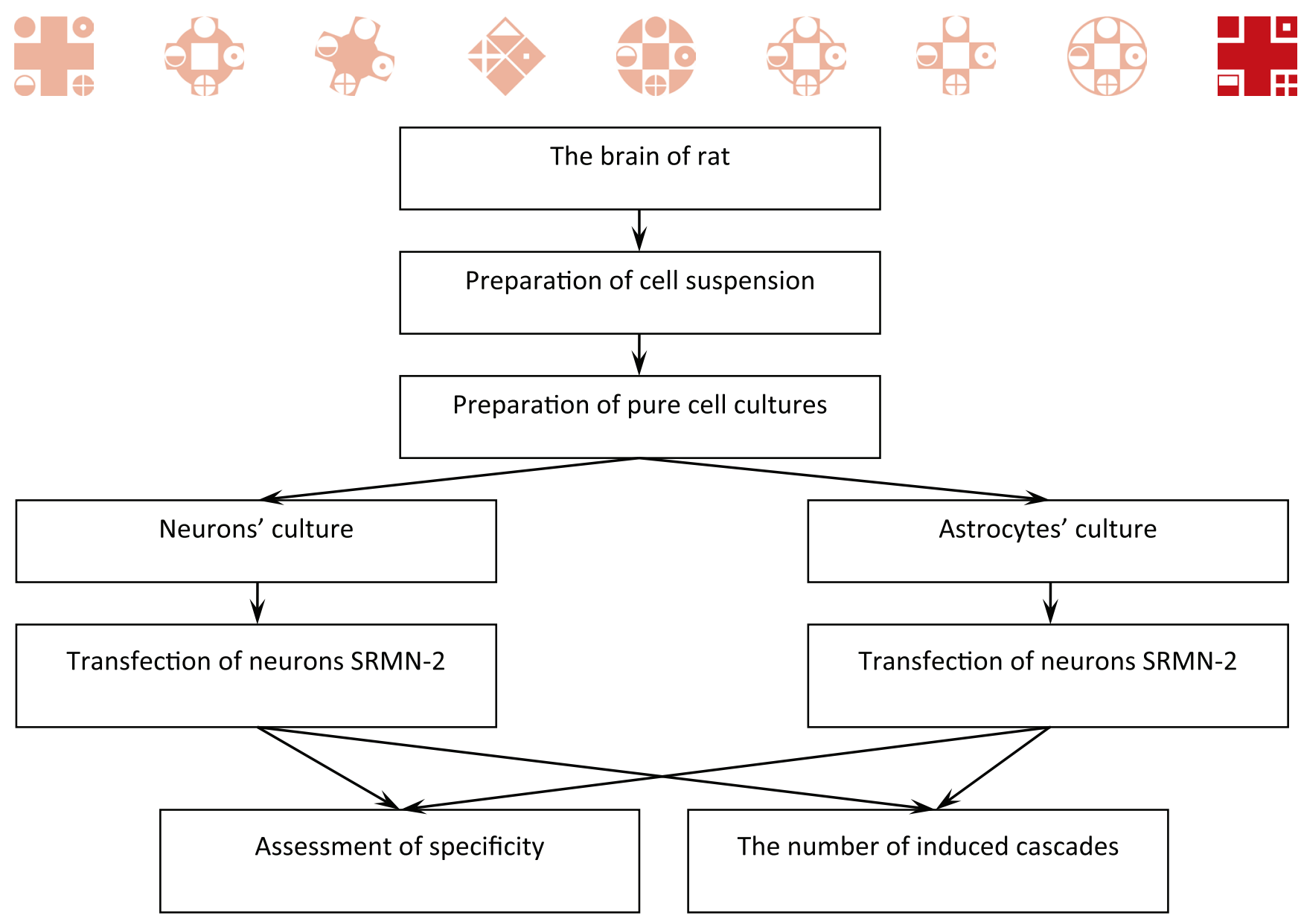

Figure 3. The plan of studying experimental SRMN samples for assessing their efficiency in the in vitro experiments

of $2.5 \times 10^{5}$ cells $/ \mathrm{cm}^{2}$. BME medium was replaced after 2.5 hours with the Neurobasal-A medium, containing $2 \mathrm{mM}$ of GlutaMAX-I, component B-27 and gentamicin (100 $\mu \mathrm{g} /$ $\mathrm{ml})$. Primary neuron cultures were incubated for 5 days in a humidified incubator at $37^{\circ} \mathrm{C}$ in an atmosphere of $5 \% \mathrm{CO}_{2}$ and $95 \%$ air.

Immunohistochemistry was performed. Cell cultures were fixed in $4 \%$ paraformaldehyde in phosphate buffer ( $\mathrm{pH}$ 7.4) for 2 hours at room temperature or 6 hours at $4^{\circ} \mathrm{C}$. Fixed cultures were washed in phosphate buffer for $15 \mathrm{~min}$ at least three times and incubated in a $10 \%$ solution of bovine serum for at least 6 hours at $4^{\circ} \mathrm{C}$. Cultures were incubated for at least 24 hours with primary antibodies to the astrocytic marker GFAP and neuronal marker NeuN (1:1000). Cultures were washed in phosphate buffer containing $0.1 \%$ bovine serum and $0.1 \%$ TritonX detergent. Cultures were incubated with secondary antibodies conjugated with fluorescent markers for at least 6 hours (1:1000). Cultures were washed in phosphate buffer for 15 minutes at least three times, stained with DAPI, and examined under an LSM 760 laser scanning confocal microscope (Zeiss, Germany).

Figures 4-5 show astrocytes and neurons transfected in vitro with genetic constructs (green) and immunohistochemically stained for the astrocyte-specific marker GFAP (red) and the neuron-specific marker NeuN (red).

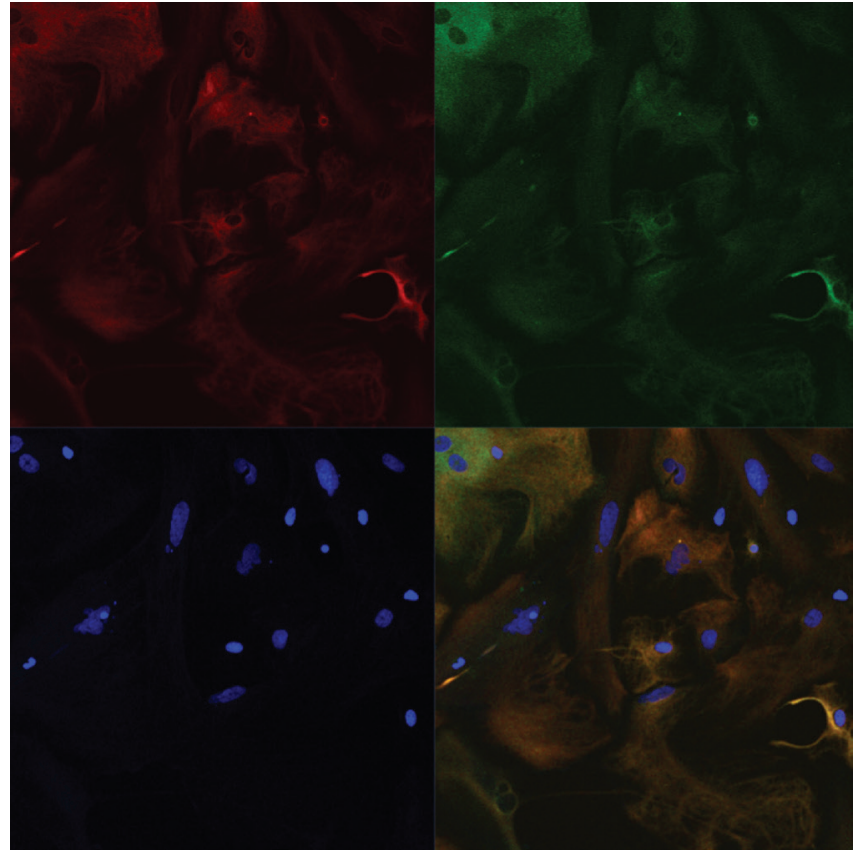

Figure 4. Astrocytes transfected with LVV-GFAP-GCaMP3 (green) and stained for the astrocyte-specific marker GFAP (red), in vitro

Colocalization of fluorescent signals produced yellow coloration at the cross-section, which allowed for the 


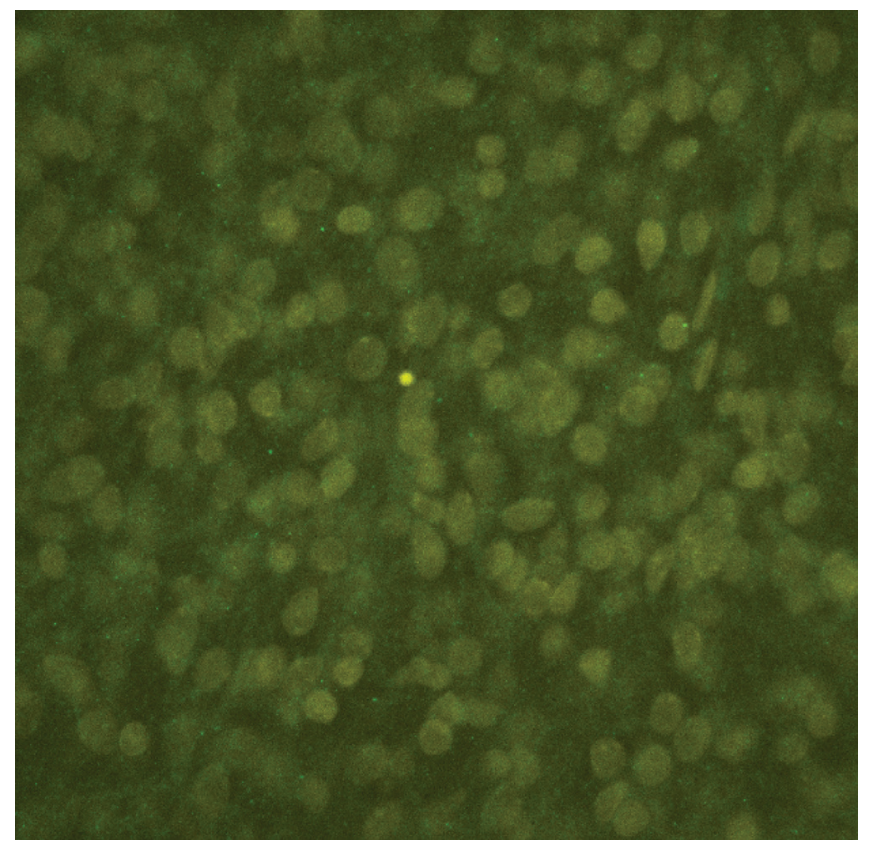

Figure 5. Neurons transfected with LVV-GFAP-TN-XXL (green) and stained for the neuron-specific marker NeuN, in vitro

quantification of cells stained only for GFAP or NeuN and cells with colocalized fluorescent signals. The specificity of the genetic construct to a particular cell type was calculated based on the number of cells per brain section that contained colocalized fluorescent signals.

The number of induced signalling cascades during SRMN-1 and SRMN-2 transfection was determined via activation of intracellular signalling cascades of neurons and astrocytes and recording of changes in fluorescence signal levels. Induced cascades were purine (purinergic) receptors $\mathrm{P} 2 \mathrm{X}$ and $\mathrm{P} 2 \mathrm{Y}$, and $\beta$-adrenergic receptors. Therefore, three ingsignalling cascades were activated: $\mathrm{X}$ - and Y-type purine receptors and $\beta$-adrenergic receptors.

Substance-activator was added to induce signal cascades of SRMN-1 and SRMN-2 transfection in pure astrocyte and neuron cultures, and the concentration of intracellular $\mathrm{Sa}^{2+}$ was measured using fluorescence. Fluorescence levels were compared to levels prior to addition of the activator. Transfected cells that exhibited a two-fold or greater increase in fluorescence intensity after addition of the appropriate activator were considered activated.

Neurons and astrocytes were grown on cover glasses and transferred with genetic constructs for expression of LVV-PRSx8-TN-XXL and LVV-GFAP-GCaMP3, respectively. Growth media Neurobasal-A (neurons) and DMEM (astrocytes) was removed using fully balanced Hanks solution (HBSS) at room temperature, and cells were transferred to the experimental cell. Cells were undisturbed for 30 minutes at room temperature. The temperature of the HBSS solution was increased to $37^{\circ} \mathrm{C}$ immediately before the experiment, and the experimental cells were transferred to the specimen stage of a laser scanning confocal microscope. Fluorescence excitation of lasers and light filters were adjusted to record fluorescence (argon laser 488 $\mathrm{nm}$, intensity $1 \% ; 510-525 \mathrm{~nm}$ ). The primary method of imaging activation of purinergic receptors was intracellular $\mathrm{Ca}^{2+}$ concentration. The following activators were used in pure cultures of astrocytes and neurons: $10 \mu \mathrm{M}$ of 2-MeSATP was added to activate P2Y-dependent $\mathrm{Ca}^{2+}$ cascades; $10 \mu \mathrm{M}$ a,b-mATP was used to activate P2Xdependent $\mathrm{Ca}^{2+}$ cascades; and $50 \mu \mathrm{M}$ of isoproterenol was used to activate $\beta$-adrenergic-dependent $\mathrm{Ca}^{2+}$ cascades. Changes in fluorescence were recorded using an LSM 780 confocal microscope (Carl Zeiss).

The experimental design of SRMN sample efficiency in vivo included stereotactic injections into rat brains, postsurgical monitoring, preparation of brain slices expressing SRMN areas, and an assessment of transfection efficiency of SRMN using laser scanning confocal microscopy. The same methods described above (in vitro studies) were used to define the specificity and number of induced cascades during SRMN-1 and SRMN-2 transfection in vivo (Fig. 6).

Specificity for a certain cell type of each genetic construct was determined using in vivo transfection of rat brain cells, obtaining brain slices, followed by staining using immunohistochemistry for cell-specific markers (same antibodies as in vitro), and recording of fluorescent signals using an LSM 780 confocal microscope (Carl Zeiss).

Viral particles were injected into the brain according to the following protocol. The solution for brain preparation contained (in $\mathrm{mM}$ ) $\mathrm{NaCl} 87, \mathrm{KCl} \mathrm{2.5,} \mathrm{MgSO}_{4} 8.48$, $\mathrm{NaH}_{2} \mathrm{PO}_{4} 1.24, \mathrm{NaHCO}_{3} 26.2, \mathrm{CaCl}_{2} 0.5$, and D-glucose 11, osmolarity (289-296 mmol/ $\mathrm{kg}$ ) and $\mathrm{pH}(7.4)$. The solution for slice incubation contained (in $\mathrm{mM}$ ) $\mathrm{NaCl} 119 ; \mathrm{KCl}, 2.5$; $\mathrm{MgSO}_{4}$ 1.3; $\mathrm{NaH}_{2} \mathrm{PO}_{4} 1 ; \mathrm{NaHCO}_{3} 26.2 ; \mathrm{CaCl}_{2} 1 ; \mathrm{MgCl}_{2}$ to 1.6; D-glucose 11; osmolarity (289-296 mmol/ $\mathrm{kg}$ ) and $\mathrm{pH}$ (7.4). The working Ringer's solution contained (in $\mathrm{mM}$ ): $\mathrm{NaCl} 119 ; \mathrm{KCl}, 2.5 ; \mathrm{MgSO}_{4} 1.3 ; \mathrm{NaH}_{2} \mathrm{PO}_{4} 1 ; \mathrm{NaHCO}_{3} 26.2$; $\mathrm{CaCl}_{2}$ 2; D-glucose 11 ( $\mathrm{pH} 7.4$; osmolarity 295 mOsm). The rat was anaesthetized and fixed in a stereotaxic frame. A cannula hole was opened, and the needle of the adapter of the microdoser was inserted into the hole.. The solution was injected (GKKI lentiviral genetic structures - LVVPRSx8-TN-XXL and LVV-GFAP-GCaMP3). Viral particles were introduced slowly at a rate not greater than $10 \mu \mathrm{l}$ within 1 minute. The needle was removed from the cannula, and the hole was closed. Animals received post-surgical care. Rats were decapitated after the recovery period, and brains were isolated and immediately transferred to the preparation solution $\left(4^{\circ} \mathrm{C}\right)$. Slices $(40$ - to $50-\mu \mathrm{m}$ thick) were prepared. Slices were taken from the bath of the vibratome using a brush and placed in carbogenized solution for incubation at $36^{\circ} \mathrm{C}$. Slices were placed under a Zeiss 780 confocal microscope (Zeiss, Germany). Brain slices in Ringer's solution were placed in a chamber with specific gas and temperature conditions, which were adjusted using Zen software (Zeiss, Germany). Sharp slices were fixed in $4 \%$ paraformaldehyde in phosphate buffer $(\mathrm{pH}$ 7.4) for 2 hours at room temperature. Subsequent processing was the same as the in vitro studies. Processing ended with laser scanning confocal microscopy. 


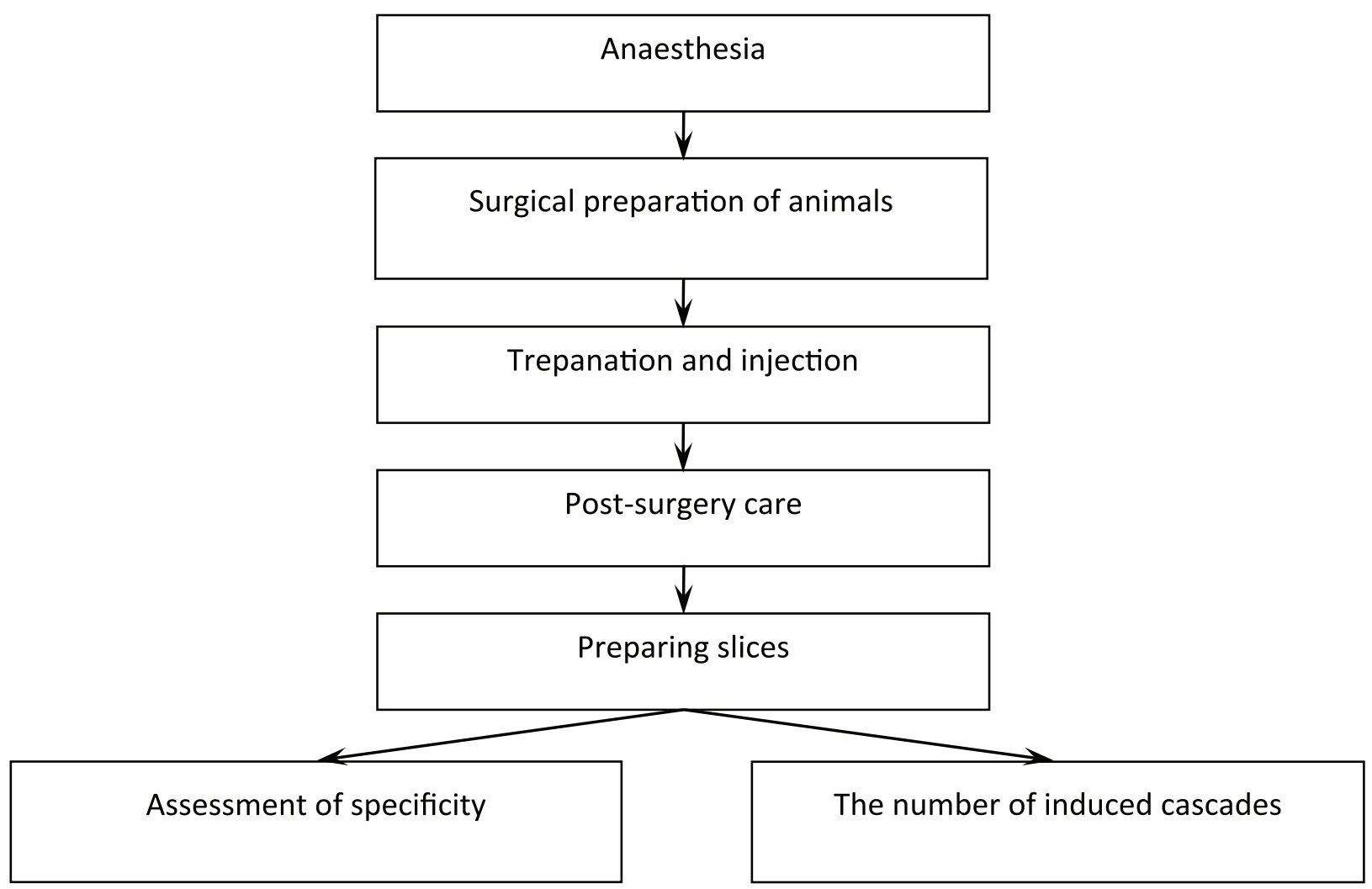

Figure 6. The plan of studying experimental SRMN samples for assessing their efficiency in the in vivo experiments

\section{Results of the in vitro studies}

Cells stained only for GFAP (astrocytes) or NeuN (neurons) and cells with colocalized fluorescent signals were counted. All genetic constructs withstood the experimental processing because fluorescent signals were registered from neurons and astrocytes transfected with a certain construct. The specificity of the genetic constructs for a certain cell type was greater than $97 \%$ for LVV-PRSx8-TN-XXL and LVV-GFAP-GCaMP3 (Table 1). Notably, the number of astrocytes was higher on average than the number of neurons.
The dynamics of calcium activity in astrocytes (Fig. 7) and neurons (Fig. 8) transfected in vitro with lentiviral vector LVV-GFAP-GCaMP3 and LVV-PRSx8-TN-XXL, respectively, in response to activation of $\mathrm{P} 2 \mathrm{X}, \mathrm{P} 2 \mathrm{Y}$ and $\beta$-adrenergic dependent $\mathrm{Ca}^{2+}$ cascades were recorded.

All figures show the fluorescence intensity of the transfected brain cells cultures before activation of $\mathrm{Ca}^{2+}$ dependent cascades and the fluorescence intensity and the dynamics of changes after activation of these cascades. The figures show samples SRMN No. 1.2 and 2.2, and similar data were obtained for other SRMN samples.

Table 1. The results of the in vitro research with definition of the specificity of the genetic constructs to a specific type of cell

\begin{tabular}{|c|c|c|c|c|c|}
\hline \multirow[b]{2}{*}{ SRMN sample } & \multirow[b]{2}{*}{$\begin{array}{c}\text { Type of } \\
\text { transfected } \\
\text { cell in vitro }\end{array}$} & \multicolumn{3}{|c|}{ Average number of cells ( 5 culture cups and slices) } & \multirow[b]{2}{*}{$\begin{array}{l}\text { Specificity of the } \\
\text { genetic construct }\end{array}$} \\
\hline & & $\begin{array}{l}\text { Number of cells immune- } \\
\text { reactive for cell-specific } \\
\text { marker }\end{array}$ & $\begin{array}{c}\text { Number of cells } \\
\text { successfully transfected } \\
\text { with the appropriate } \\
\text { genetic design }\end{array}$ & $\begin{array}{l}\text { Number of cells immune- } \\
\text { reactive for cell-specific marker } \\
\text { and successfully transfected with } \\
\text { a genetic construct }\end{array}$ & \\
\hline No. 1.1 & \multirow{3}{*}{ astrocytes } & $415 \pm 47$ & $419 \pm 18$ & $404 \pm 26$ & $97.4 \%$ \\
\hline No. 1.2 & & $414 \pm 45$ & $420 \pm 16$ & $405 \pm 20$ & $97.1 \%$ \\
\hline No. 1.3 & & $410 \pm 27$ & $425 \pm 19$ & $400 \pm 16$ & $97.5 \%$ \\
\hline No. 2.1 & \multirow{3}{*}{ neurons } & $340 \pm 15$ & $348 \pm 34$ & $331 \pm 16$ & $97.4 \%$ \\
\hline No. 2.2 & & $329 \pm 17$ & $337 \pm 34$ & $320 \pm 10$ & $97.2 \%$ \\
\hline No. 2.3 & & $334 \pm 15$ & $343 \pm 34$ & $325 \pm 16$ & $97.3 \%$ \\
\hline
\end{tabular}




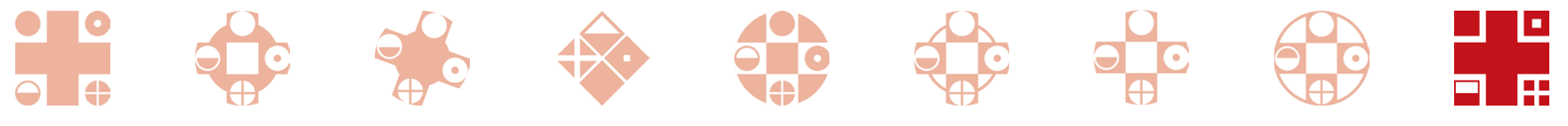
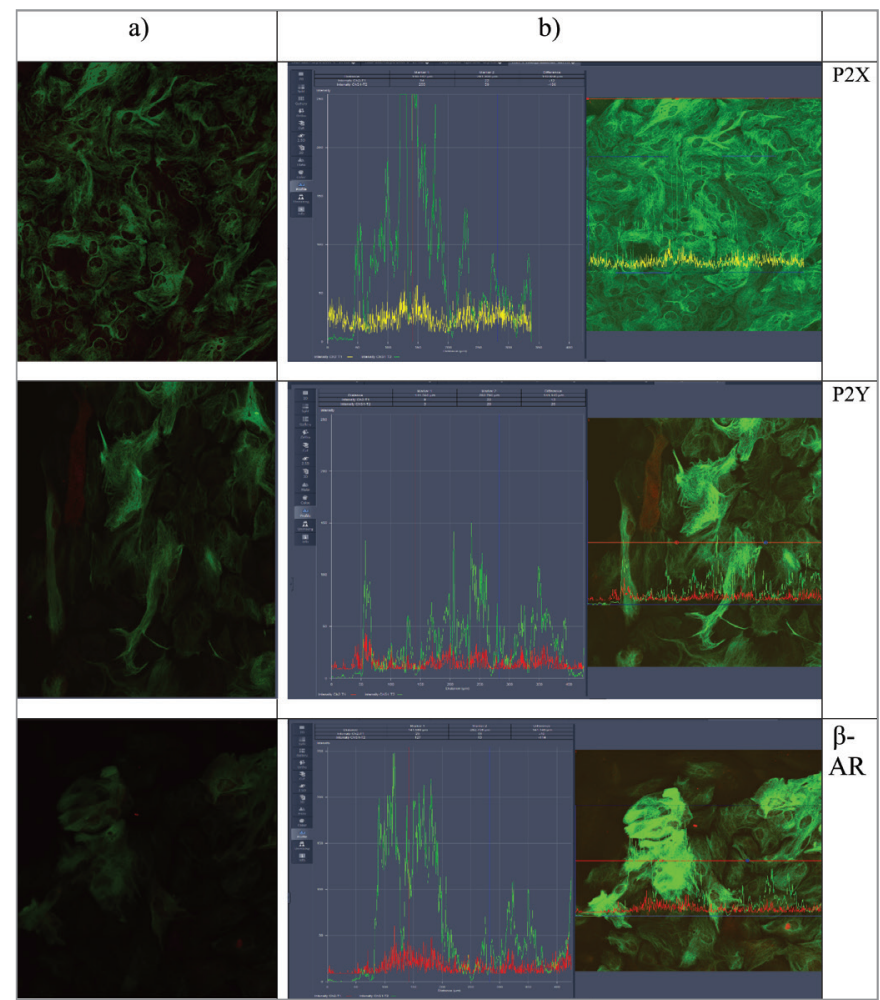

Figure 7. A pure culture of rat brain astroglia cells: a) astrocytes transfected with lentiviral vector LVV-GFAP-GCaMP3 in vitro and expressing GKKI G-CaMP3; b) astrocytes expressing GKKI G-CaMP3 when activated with P2X dependent CA2 + cascades (top), when activated with P2Y dependent $\mathrm{Ca} 2+$ cascades (middle), $\beta$-adrenergic dependent $\mathrm{Ca} 2+$ cascades (bottom).

Changes in the fluorescence intensity upwards (more than twice) confirmed the activation of intracellular signalling cascades in neurons and astrocytes. The increase in the fluorescence intensity in astrocytes was slightly higher than neurons.
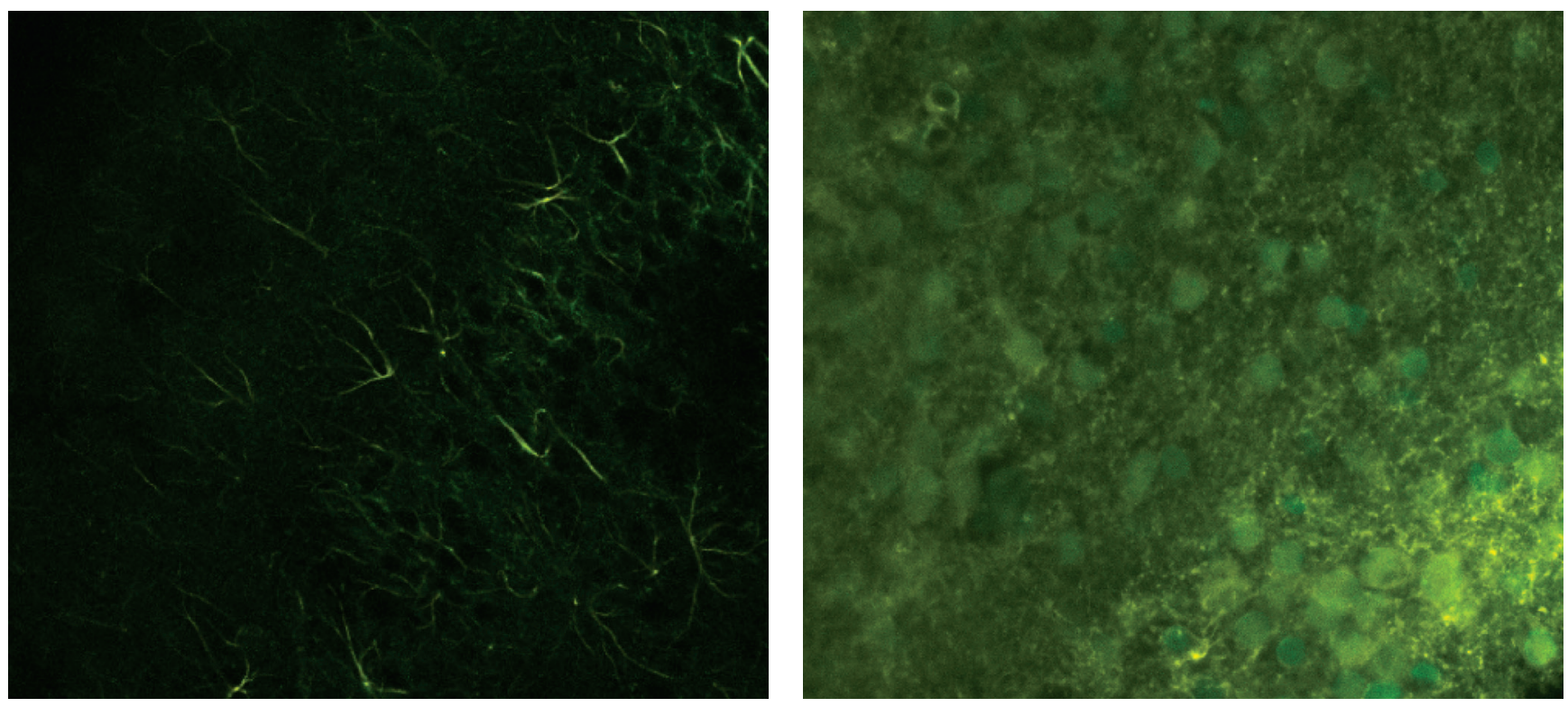

Figure 9. Astrocytes transfected with LVV-GFAP-GCaMP3 (green) and stained for the astrocyte-specific marker GFAP (red), in vivo

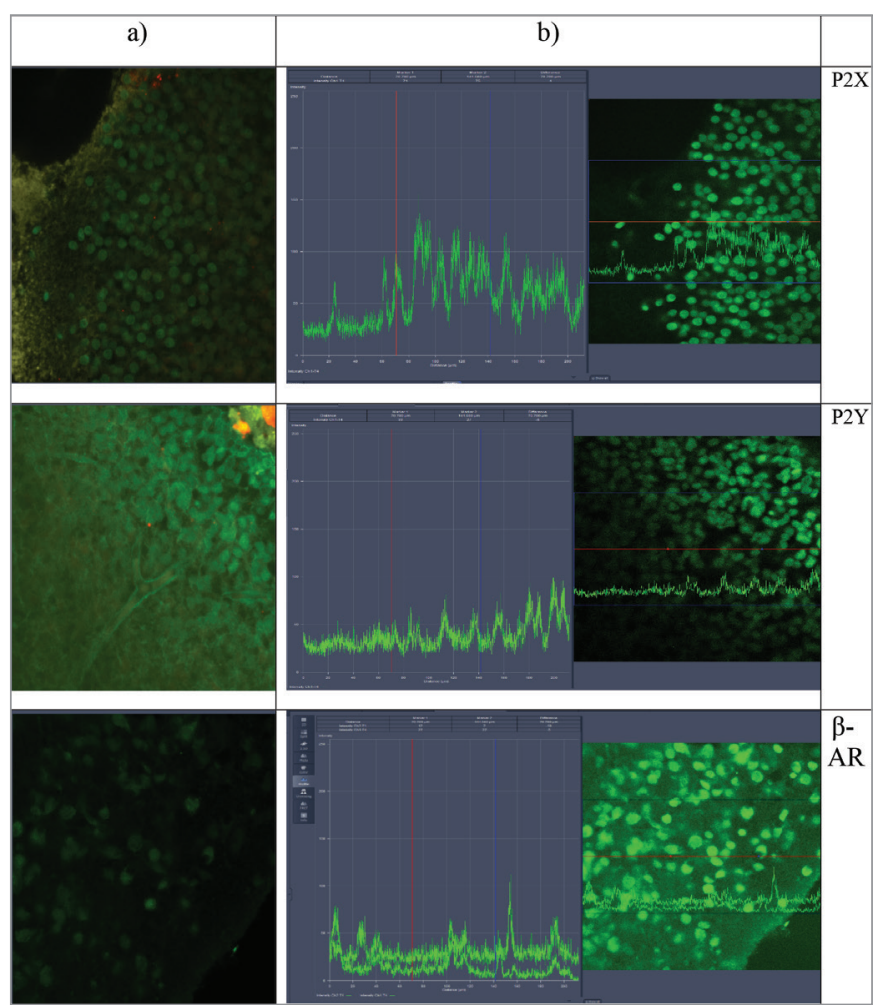

Figure 8. Pure culture of rat brain cells: a) neurons transfected with lentiviral vector LVV-PRSx8-TN-XXL in vitro and expressing GKKI TN-XXL; b) neurons expressing GKKI TN-XXL when activated with P2Y dependent CA2+ cascades (top), activated with P2Y-dependent Ca2+ cascades (middle), $\beta$-adrenergic dependent $\mathrm{Ca} 2+$ cascades (bottom).

\section{Results of the in vivo studies}

Figures 9-10 show astrocytes and neurons transfected in vivo with genetic constructs (green) and immunohistochemically stained for the astrocyte-specific marker GFAP
Figure 10. Neurons transfected with LVV-GFAP-TN-XXL (green) and stained for the neuron-specific marker NeuN (red), in vivo 
Table 2. The results of the in vivo research with definition of the specificity of the genetic constructs to a specific type of cell

\begin{tabular}{|c|c|c|c|c|c|}
\hline SRMN sample & $\begin{array}{c}\text { Type of } \\
\text { transfected cell }\end{array}$ & $\begin{array}{l}\text { Number of cells immune- } \\
\text { reactive for cell-specific } \\
\text { marker }\end{array}$ & $\begin{array}{l}\text { Number of cells } \\
\text { successfully transfected } \\
\text { with the appropriate } \\
\text { genetic design }\end{array}$ & $\begin{array}{l}\text { Number of cells immune- } \\
\text { reactive for cell-specific marker } \\
\text { and successfully transfected with } \\
\text { a genetic construct }\end{array}$ & $\begin{array}{l}\text { Specificity of the } \\
\text { genetic construct }\end{array}$ \\
\hline No. 1.1 & \multirow{3}{*}{ astrocytes } & $410 \pm 34$ & $408 \pm 18$ & $422 \pm 16$ & $97.2 \%$ \\
\hline No. 1.2 & & $400 \pm 25$ & $410 \pm 20$ & $388 \pm 14$ & $97.0 \%$ \\
\hline No. 1.3 & & $415 \pm 47$ & $414 \pm 22$ & $404 \pm 26$ & $97.3 \%$ \\
\hline No. 2.1 & \multirow{3}{*}{ neurons } & $330 \pm 16$ & $341 \pm 34$ & $321 \pm 16$ & $97.3 \%$ \\
\hline No. 2.2 & & $340 \pm 15$ & $365 \pm 42$ & $330 \pm 16$ & $97.1 \%$ \\
\hline No. 2.3 & & $323 \pm 10$ & $340 \pm 37$ & $314 \pm 16$ & $97.2 \%$ \\
\hline
\end{tabular}

(red) and the neuron-specific marker NeuN (red). Colocalization of fluorescent signals produced a yellow coloration in the section.

Cells stained only for GFAP (astrocytes) or NeuN (neurons) and cells with colocalized fluorescent signals were counted (Table 2). All genetic constructs withstood

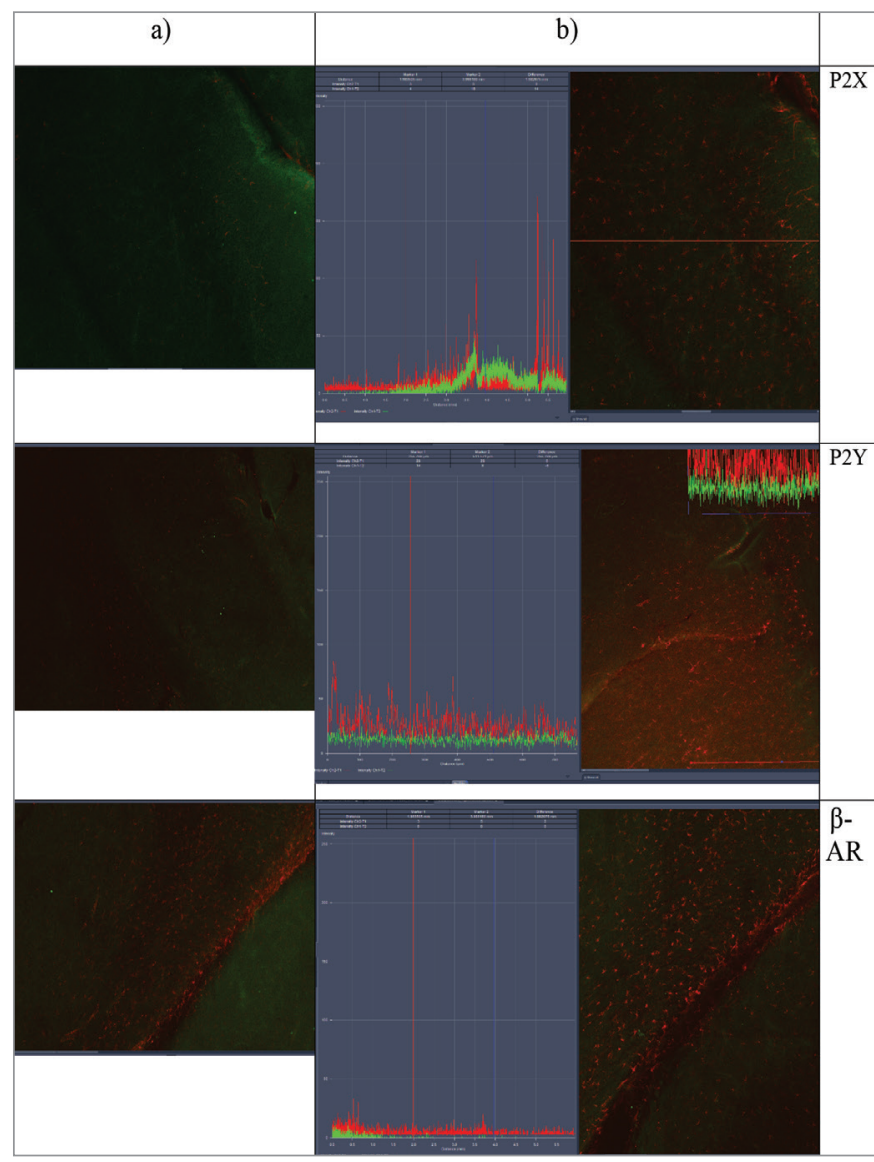

Figure 11. Organotypic rat brain slices: a) astrocytes transfected with lentiviral vector LVV-GFAP-GCaMP3 in vivo and expressing GKKI G-CaMP3; b) astrocytes expressing GKKI G-CaMP3 upon activation of P2X dependent $\mathrm{Ca}_{2}{ }^{+}$cascades (top), upon activation of P2Y dependent $\mathrm{Ca}_{2}{ }^{+}$cascades (middle), $\beta$ - adrenergic dependent $\mathrm{Ca}_{2}{ }^{+}$cascades (bottom). the experimental processing because fluorescent signals were registered from neurons and astrocytes transfected with a certain construct. The specificity of genetic constructs for a certain cell type was calculated based on the number of cells in a slice of brain with colocalized fluorescent signal that was just over $97 \%$ for all samples.

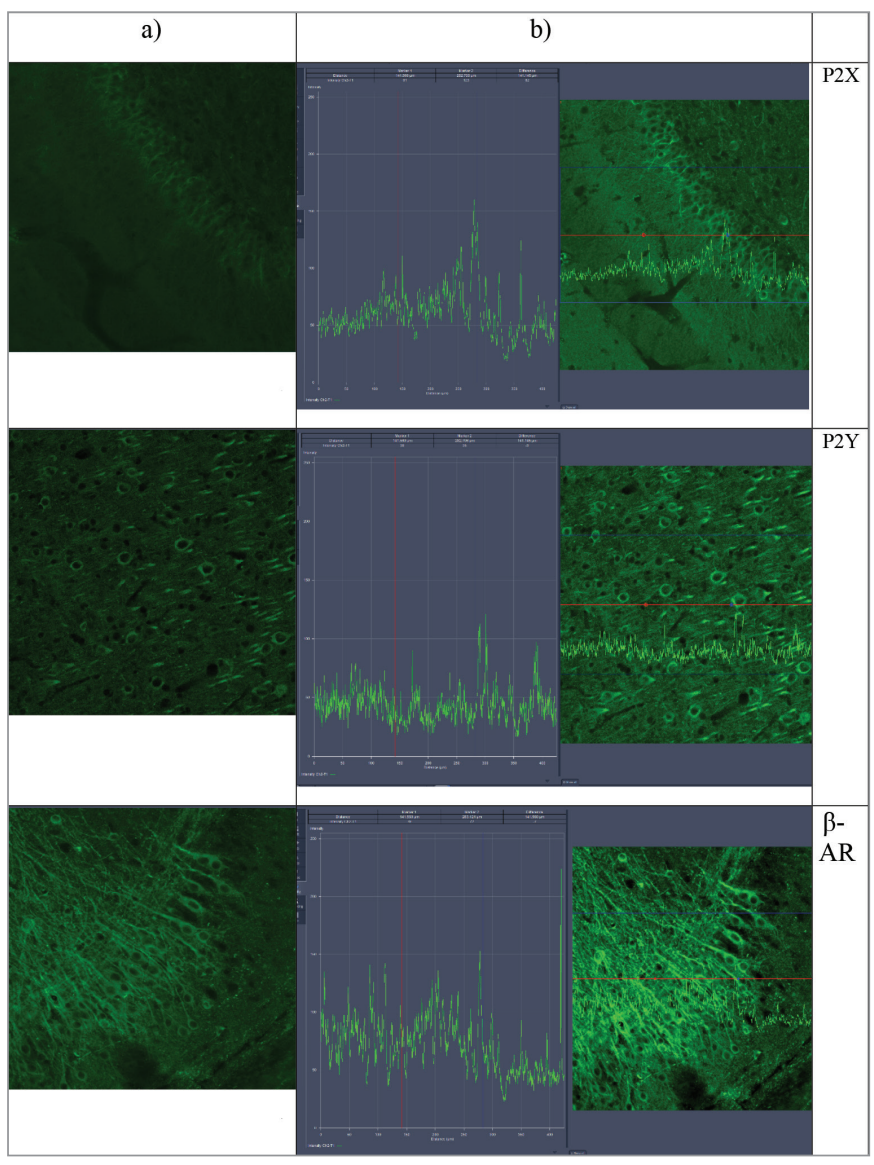

Figure 12. Organotypic rat brain slices: a) neurons transfected with lentiviral vector LVV-PRSx8-TN-XXL in vivo and expressing GKKI TN-XXL; b) neurons expressing GKKI TN-XXL upon activation of P2Y dependent $\mathrm{Ca}_{2}{ }^{+}$cascades (top), upon activation of P2Y dependent $\mathrm{Ca}_{2}{ }^{+}$cascades (middle), $\beta$ - adrenergic dependent $\mathrm{Ca} 2+$ cascades (bottom). 
The results of the in vivo experiment repeated the in vitro experiment.

The number of induced signalling cascades in SRMN-1 and SRMN-2 transfection was determined similar to the in vitro studies. The only exception was that all experiments were performed on the sharp brain slices prepared in the same manner as determinations of the specificity of the genetic structures in vivo.

The dynamics of calcium activity in astrocytes (Fig. 11) and neurons (Fig. 12) transfected with appropriate lentiviral vector in vitro in response to activation of purinergic (P2X, P2Y) and $\beta$-dependent adrenal $\mathrm{Ca}^{2+}$ cascades exhibited comparable results to the in vitro experiments. All figures show the fluorescence intensity of transfected organotypical brain cells slices before activation of cascades and the fluorescence intensity and the dynamics of changes after activation of these $\mathrm{Ca}^{2+}$-dependent cascades. All SRMN samples produced the same results.

The results revealed a significant upward change in the fluorescence intensity, which also confirmed activation of the intracellular signalling cascades in neurons and astrocytes.

\section{DISCUSSION}

Astrocytes were discovered and described approximately 150 years ago, and these cells are increasingly the subject of study in various areas. Adequate methods of detecting cells of the nervous system must be developed to implement fundamental discoveries, which was the goal of the authors of this study. The end products of the developed methods are sets of reactants for modelling neurogenesis (SRMN) containing genetic constructs based on the lentiviral vector with built-in cell-specific sequences that provide expression of these genetic structures in astrocytes (SRMN-1) or neurons (SRMN-2) in DMEM with $10 \%$ foetal bovine serum. The genetic construct successfully integrates into the genome of target cells to induce stable expression of the genetically encoded calcium indicator GCaMP3 for 48-72 hours after addition to cultures in vitro or after injection into the brain parenchyma in vivo.

The in vitro and in vivo experiments demonstrated a greater than two-fold increase in fluorescent signals in response to the addition of activators that stimulate purinergic and beta-adrenal calcium-dependent signalling cascades in various cells of the central nervous system and high specificity to a particular cell type (97\%). These results confirmed the efficiency of the developed method. The results obtained in pure cell cultures were identical to the results in animals.

An important fundamental conclusion of this work is evidence of the critical role of astrocytes in ensuring neurogenesis and neuroplasticity.

The developed method of genetic detection of neurogenesis is based on the use of a cellular-specific promoter to control the simultaneous expression of the required transgene and strong artificial activator of transcription to enhance transcription via binding to specific binding sites introduced into the sequence of the promoter. Therefore, two cell cycle-specific promoters are used: one for transcription of the transgene of interest, and the other for the expression of the transactivator.

The results of this method of assessing the efficiency of experimental SRMN samples revealed the successful and stable transfection of catecholaminergic neurons and astrocytes, experimental data on transfection efficiency, the specificity of the developed genetic structures as part of SRMN, and calcium dynamics in transfected neurons and astrocytes.

The use of this method allows cell- and phenotype-specific transfection of various types of neurons that ensures the targeted control of intracellular cascades (13) and visualization of in vitro-transfected cells via the embedding of genetically encoded calcium indicators into the developed genetic construct.

\section{CONCLUSION}

The developed methods and sets of reactants for modelling neurogenesis are intended to investigate brain functioning, particularly neural networks of mammalian brains using analyses of calcium dynamics in catecholaminergic neurons and astrocytes. These methods allow investigations of the functional status of brain cells in the normal state and in various pathologies. The scope of this methodology application is limited to fundamental and scientific research in neurosciences and molecular biology, particularly the molecular mechanisms of various diseases of the central nervous system. However, this development may become a routine method to monitor the functional state of neurons and astrocytes in vivo to examine developed medicines and monitor the efficiency of new methods for the treatment of schizophrenia, neurodegeneration, cerebrovascular, demyelination, and other highly disabling and socially significant diseases.

Notably, this technology based on cell viral transfection provides the possibility of non-invasive application, of which the best use is chemogenetic expression monitoring. This technique achieves specificity of expression of incorporated genes in certain brain areas. Therefore, our and similar developments may be introduced into clinical practice in the future to create a new type of gene therapy for diseases that cause degradation of neurons, astrocytes and other brain cells.

\section{ACKNOWLEDGMENTS}

This work was supported by the Ministry of Education and Science of the Russian Federation grant (FCP No. 14.575.21.0074 (unique identifier - RFMEFI57514X0074) in the Immanuel Kant Baltic Federal University. 


\section{REFERENCES}

1. Erkkinen M.G., Kim M.O., Geschwind M.D. Clinical Neurology and Epidemiology of the Major Neurodegenerative Diseases. Cold Spring Harb Perspect Biol. 2017; (17): a033118.

2. Rumyantseva S.A., Silina E.V., Svischeva S.R., Komarov A.N. Medical and organizational issues of pre- and post-stroke disablement. Zhurnal Nevrologii i Psihiatrii imeni S.S. Korsakova. 2013;9:43-49.

3. Gomazkov O.A., Afanasiev V.V., Rumyantseva S.A., Stupin V.A., Silina E.V., Sokhova O.A. Current concepts of neurocytoprotective therapy. Neuroscience and Behavioral Physiology, 2013;3(43):374-379.

4. Verkhratsky A. Sofroniew M., Messing A. et al. Neurological diseases as primary gliopathies: a reassessment of neurocentrism. ASN Neuro. 2012;4:e00082. https:// www.ncbi.nlm.nih.gov/pmc/articles/PMC3320215/

5. Khakh B.S., Sofroniew M.V. Diversity of astrocyte functions and phenotypes in neural circuits. Nat Neurosci. 2015;18:942-952.

6. Gray M.T., Woulfe J.M. Striatal blood-brain barrier permeability in Parkinson's disease. J. Cereb. Blood Flow Metab. 2015;35:747-750.
7. Herculano-Houzel S. The glia/neuron ratio: how it varies uniformly across brain structures and species and what that means for brain physiology and evolution. Glia. 2014;62:1377-1391.

8. Haydon PG, Nedergaard M. How do astrocytes participate in neural plasticity? Cold Spring Harb Perspect Biol. 2014;11;7(3):a020438.

9. Zhang Y., Sloan S. A., Clarke L. E., Caneda C., Plaza C. A., Blumenthal P. D., et al. Purification and characterization of progenitor and mature human astrocytes reveals transcriptional and functional differences with mouse. Neuron. 2016;89:37-53.

10. Sofroniew M.V., Vinters H.V. Astrocytes: biology and pathology. Acta Neuropathol. 2010;119:7-35.

11. Zuchero J. B., Barres B. A. Glia in mammalian development and disease. Development 2015;142:3805-3809.

12. Booth H., Hirst W., Wade-Martins R. The Role of Astrocyte Dysfunction in Parkinson's Disease Pathogenesis. Trends Neurosci. 2017; 40(6): 358-370.

13. Kasymov V.A., Bogdanov E.A., Silina E.V., Litvitsky P.F., Shusharina N.N., Patrushev M.V. Evaluation of the functional status of signal systems of astrocytes by the developed reagent kit basedon genetic constructions (experimental study). Asian Journal of Pharmaceutics. 2017;11 (4):787-793. 